\title{
Catherine Sauviat, Laurence Lizé, La crise du modèle social américain
}

Rennes, Presses universitaires de Rennes, coll. « Économie et société », 2010

\section{Thierry Kirat}

\section{OpenEdition \\ Journals}

Édition électronique

URL : http://journals.openedition.org/travailemploi/5761

DOI : 10.4000/travailemploi.5761

ISSN : 1775-416X

Éditeur

DARES - Ministère du Travail

Édition imprimée

Date de publication : 15 septembre 2012

Pagination : 139-140

ISSN : 0224-4365

Référence électronique

Thierry Kirat, "Catherine Sauviat, Laurence Lizé, La crise du modèle social américain », Travail et Emploi [En ligne], 131 | 2012, mis en ligne le 01 décembre 2012, consulté le 22 septembre 2020. URL : http:// journals.openedition.org/travailemploi/5761; DOI : https://doi.org/10.4000/travailemploi.5761

Ce document a été généré automatiquement le 22 septembre 2020.

(C) Direction de l'animation de la recherche, des études et des statistiques (Dares) 


\section{Catherine Sauviat, Laurence Lizé, La crise du modèle social américain}

Rennes, Presses universitaires de Rennes, coll. « Économie et société », 2010

\section{Thierry Kirat}

\section{RÉFÉRENCE}

Catherine Sauviat, Laurence Lizé, La crise du modèle social américain, Rennes, Presses universitaires de Rennes, coll. «Économie et société », 2010, 230 p.

1 Dans leur ouvrage, les auteures offrent une belle mise en perspective du modèle social américain et une analyse minutieuse de sa construction, de ses évolutions et de la crise qui le caractérise. Catherine Sauviat et Laurence Lizé définissent un modèle social comme « un ensemble de valeurs et d'institutions clés sous-tendant un certain type d'organisation de l'économie et de la société et les objectifs qu'un pays se fixe dans le long terme dans ce domaine » (p. 1).

Le livre est divisé en quatre parties inégales en nombres de pages. Une première partie présente les principales institutions du marché du travail et leur érosion. Elle met l'accent sur l'existence d'une législation peu étoffée, la fragmentation des institutions de la relation d'emploi (chapitre 1), le rétrécissement du champ de la négociation collective et le déclin syndical (chapitre 2), la perte d'efficacité du salaire minimum (chapitre 3) avant de montrer combien le système d'assurance chômage est minimaliste (chapitre 4). Une deuxième partie argumente l'idée selon laquelle les performances du marché du travail américain sont en trompe-l'œil, déclinant plusieurs arguments : le premier porte sur la mesure du chômage (chapitre 5) ; le second sur l'imputation des bonnes performances du marché du travail à sa flexibilité plutôt qu'à la croissance tirée par la consommation privée (et l'endettement des ménages) et l'investissement (chapitre 6); le troisième sur la dualisation du marché du travail et la montée en puissance des emplois atypiques (chapitre 7). Le dernier argument porte sur les 
politiques de l'emploi, qui s'avèrent essentiellement passives, en cohérence à la fois avec le libéralisme économique et la domination intellectuelle du modèle d'offre de travail. La troisième partie de l'ouvrage traite, en deux chapitres, de la crise du « Welfare capitalism »: un système de santé coûteux et inéquitable (chapitre 9) et un système de retraites complémentaires démantelé (chapitre 10). La quatrième partie aborde enfin la croissance des inégalités, en partant du «mystère de la quasi-stabilité du partage de la valeur ajoutée » (chapitre 11), avant de décrire le creusement des inégalités de revenus dans les années 1980 (chapitre 12), le développement de la pauvreté qui touche les jeunes, les inactifs et les travailleurs à bas salaire (chapitre 13) et d'analyser les faibles performances des politiques de redistribution et de lutte contre la pauvreté (chapitre 14).

3 Cet ouvrage est appelé à servir de référence sur le sujet. Il conjugue avec succès l'analyse économique, la sociologie des relations professionnelles et une perspective historique. Il ne s'agit ni d'un essai, ni d'un ouvrage de vulgarisation qui résumerait pour le grand public les analyses économiques techniquement complexes, mais d'un ouvrage d'économie politique savant, informé et analytique, qui tisse une toile avec les relations professionnelles, les acteurs et leur contribution par exemple à la construction du régime de retraites, et une perspective longue attentive à l'émergence des institutions. Ajoutons à cela une dimension politique qui est loin d'être mineure dans l'ouvrage. Les auteures font l'économie d'une présentation de modèles microéconométriques reposant sur des faits stylisés ou des caractérisations idéal-typiques de situations : Catherine Sauviat et Laurence Lizé donnent corps, à partir de statistiques descriptives, à une économie des politiques sociales, des syndicats, de l'État fédéral et des États fédérés, qui relève d'une démarche institutionnelle et historique. Elle conduit à privilégier, lorsque cela est nécessaire, la logique du sens des évaluations économiques réalisées par les institutions publiques (notamment le Congrès et le Department of Labor [équivalent du ministère du Travail français]) ou pour leur compte sur les dispositifs de politique économique et sociale.

4 L'argumentation est soutenue par de nombreuses données statistiques et des graphiques judicieusement placés dans le texte et commentés. La réflexion conduite sur les catégories de travailleurs (notamment celle d'employee) et les effectifs de salariés bénéficiaires de dispositifs sociaux (amélioration des conditions de travail, règles de licenciement, droit à la santé ou à la retraite) mérite d'être signalée et saluée. Il est particulièrement important d'avoir en mémoire le fait qu'un certain nombre de protections - issues pour l'essentiel du New Deal - relatives aux conditions de travail et de rémunération découlent de la reconnaissance de la qualité d'employee par l'employeur, sous un contrôle peu efficace du ministère du travail, ce qui de fait permet de déclarer des salariés comme travailleurs indépendants plutôt que comme employees ; cette pratique permet alors d'échapper à une partie des cotisations sociales et obligations relatives à la durée du travail et aux salaires (pp. 31 et 32).

5 Les développements consacrés dans la première partie à la transformation du régime de protection de l'emploi sont d'un grand intérêt. Ils confortent la thèse du déclin des relations collectives de travail, des syndicats, des négociations collectives (labor law) au profit d'institutions centrées sur les droits individuels (employment law). Ainsi, ont été mis progressivement au premier plan les droits de la personne avec un important arsenal législatif et jurisprudentiel de lutte contre les discriminations au travail, dont découle une "judiciarisation des droits» (sur laquelle les auteures ne s'attardent pas, 
ce que l'on peut regretter). Au titre des questions importantes sur lesquelles le lecteur aurait aimé trouver davantage de développements, on peut évoquer la question des controverses sur les effets du salaire minimum. Les auteures abordent assez rapidement (pp. 67-69) cette question sur le plan académique. Il aurait été intéressant de donner davantage de précisions sur les institutions de recherche et les think-tanks impliqués dans ces débats.

6 Si les transformations du «modèle social américain » sont mises en rapport avec les politiques publiques et les changements institutionnels, les transformations structurelles du capitalisme américain, micro comme macro-économiques, restent assez peu explorées. Ainsi, la financiarisation du gouvernement d'entreprise n'est que mentionnée (p.194); les transformations macroéconomiques du régime d'accumulation sont réduites à la mort du fordisme, ce qui constitue un argument certes acceptable, mais un peu court. Il en va de même avec le « régime institutionnel de la politique macroéconomique " évoqué par les auteures. Sur le plan formel, on pourra regretter quelques défauts de présentation, tels des graphiques dont l'échelle n'est pas toujours précisée ainsi que l'absence de conclusion générale au terme de chaque partie.

7 Ces quelques éléments de discussion critique de l'ouvrage de Catherine Sauviat et Laurence Lizé n'enlèvent rien à ses qualités : l'ouvrage est riche et dense; le lecteur y trouvera des analyses claires, bien écrites, documentées, qui relèvent d'une perspective d'économie politique convaincante et brillante.

\section{AUTEURS}

\section{THIERRY KIRAT}

Institut de recherche interdisciplinaire en sciences sociales (IRISSO) - Université Paris Dauphine 